\title{
A Novel Protein Com1 Is Required for Normal Conidium Morphology and Full Virulence in Magnaporthe oryzae
}

\author{
Jun Yang, ${ }^{1}$ Xiaoyan Zhao,, Jing Sun, ${ }^{1}$ Zhensheng Kang, ${ }^{2}$ Shengli Ding, ${ }^{3}$ Jin-Rong $\mathrm{Xu},{ }^{3}$ and \\ You-Liang Peng ${ }^{1,4}$ \\ ${ }^{1}$ State Key Laboratory of Agrobiotechnology and MOA Key Laboratory of Plant Pathology, China Agricultural University, \\ Beijing 100193, China; ${ }^{2}$ College of Plant Protection, Northwest A\&F University, Yangling, Shaanxi 712100, China; \\ ${ }^{3}$ Department of Botany and Plant Pathology, Purdue University, West Lafayette, IN 47907, USA; ${ }^{4}$ National Plant Gene \\ Research Center (Beijing), Beijing 100193, China
}

Submitted 14 March 2009. Accepted 16 September 2009.

In Magnaporthe oryzae, pyriform conidia are the primary inoculum and the main source for disease dissemination in the field. In this study, we identified and characterized the COM1 gene that was disrupted in three insertional mutants producing slender conidia. COM1 encodes a putative transcription regulator unique to filamentous ascomycetes. The com1 disruption and deletion mutants had similar defects in conidium morphology and were significantly reduced in virulence on rice and barley seedlings. Microscopic examination revealed that the $\Delta c o m 1$ mutants were defective in appressorium turgor generation, penetration, and infectious growth. COM1 was expressed constitutively in M. oryzae. The Com1 protein had putative helix-loop-helix structures and three predicted nuclear localization signal sequences. In transformants expressing $C O M 1^{335-613}$-enhanced green fluorescent protein fusion constructs, fluorescence signals were observed in the nucleus. Our data indicated that the COM1 gene may encode a novel transcription regulator that regulates conidial development and invasive growth in M. oryzae.

The heterothallic, haploid ascomycete Magnaporthe oryzae is pathogenic to important crops such as rice and wheat. Rice blast caused by this fungus is one of the most destructive fungal diseases of rice worldwide (Valent et al. 1991; Talbot 2003). Rice remains the major food crop for more than a third of the world's population (Ford et al. 1994); therefore, rice blast is recognized as one of the main pathological threats to world food supplies. M. oryzae is amenable to both classical and molecular genetic manipulations. In the past decade, rice blast has been developed as a model system to study fungusplant interactions (Valent 1990; Talbot 2003; Dean et al. 2005; Xu et al. 2007).

The $M$. oryzae infection cycle is initiated by the attachment of three-celled conidia to the rice leaf surface. The preformed spore tip mucilage (STM) of M. oryzae is stored in the apical cell and released upon contact with the plant surface (Hamer et

Corresponding author: Y. -L. Peng; Telephone: +86-10-6273-2541; Fax: +86-10-6273-3607; E-mail: pengyl@ cau.edu.cn

J. Yang and X. Zhao contributed equally to this study.

* The $e$-Xtra logo stands for "electronic extra" and indicates that two supplemental figures are published online. al. 1988). Conidia germinate immediately after attachment and germ tubes differentiate into specialized infection structures called appressoria. The fungus uses the enormous turgor pressure generated in appressoria for plant penetration (Howard et al. 1991). After penetration, infectious hyphae initially grow biotrophically in plant cells (Kankanala et al. 2007). Eventually, the plant cells are killed and lesions develop during invasive growth of the fungus, which can release thousands of conidia from each lesion. Many environmental cues, including moisture, temperature, and light, are known to affect conidiation in M. oryzae (Pinnschmidt et al. 1995). Considering the role of conidia in the disease cycle of rice blast, the morphology of pyriform conidia may be important for spore dispersal and the initiation of plant infection.

To date, several mutants with altered conidium morphology, including smo, con 1, con 2 , con 4, con 7, acrl, and chm1, have been identified by chemical or insertional mutagenesis (Hamer et al. 1989; Shi and Leung 1995; Lau and Hamer 1998; Li et al. 2004). Conidiation is significantly reduced ( $>90 \%$ reduction) in both conl and con 2 mutants. The conl mutant produces a terminal elongated conidium but the con 2 mutant produces mostly nonseptate or two-celled conidia. The con 4 and con 7 mutants produce conidia with abnormal cell shapes, and their conidial production is reduced by approximately $35 \%$ (Shi and Leung 1995). CON7 encodes a transcription factor with a zinc finger motif and a nuclear localization signal (Odenbach et al. 2007). The chml mutant, like the con $7 \mathrm{mu}-$ tant, produces conidia that are often misshapen and two-celled, with defects in the basal scar area (Li et al. 2004). The acrl mutant was first isolated as a restriction-enzyme-mediated DNA integration (REMI) mutant that is reduced in pathogenicity (Lau and Hamer 1998; Nishimura et al. 2000). Deletion or disruption of $A C R I$ results in the production of head-totail (acropetal) arrays of elongated conidia. The ACRl gene seems to be a stage-specific negative regulator of conidiation required to establish a sympodial pattern of conidiation (Lau and Hamer 1998). It is homologous to the medA gene that regulates conidiation in Aspergillus nidulans (Yu et al. 2006).

In this study, we isolated several $M$. oryzae mutants that produced slender conidia by a large-scale REMI mutagenesis. Three of these mutants were disrupted in the conidium morphology mutant 1 (COM1) gene. The $\Delta$ coml mutants were significantly reduced in virulence on rice and barley seedlings. $C O M 1$ was constitutively expressed in $M$. oryzae and required for appressorium turgor generation and plant penetration. 


\section{RESULTS}

Isolation of mutants with altered conidium morphology.

To identify genes that are important for development and pathogenesis in $M$. oryzae, we used the REMI approach to generate a collection of hygromycin-resistant transformants of the wild-type strain P131 (Peng and Shishiyama 1988; Sweigard et al. 1998). Five mutants that formed slender conidia, H3035, H3587, K2877, S1005, and X54, were identified after screening 12,590 transformants. On average, conidia produced by these five mutants were approximately $25 \%$ longer than those of P131 (Table 1). However, in comparison with wild-type conidia, the widths of the basal and middle conidial cells of these mutants were reduced (Table 1). Mutant conidia also tended to have wider appendages at the basal cell (Fig. 1A).
Table 1. Lengths and widths of conidia produced by the wild-type and mutant strains of Magnaporthe oryzae

\begin{tabular}{|c|c|c|}
\hline Strains & Length $(\mu \mathrm{m})^{\mathbf{a}}$ & Width $(\mu \mathrm{m})^{\mathrm{b}}$ \\
\hline P131 (wild-type) & $23.4 \pm 1.9$ & $9.7 \pm 0.5$ \\
\hline $\mathrm{X} 54(\mathrm{com} 1)$ & $29.5 \pm 2.2$ & $8.2 \pm 0.9$ \\
\hline $\mathrm{H} 3035$ (com1) & $29.0 \pm 2.1$ & $8.1 \pm 0.8$ \\
\hline $\mathrm{H} 3587$ (coml) & $29.5 \pm 2.3$ & $8.0 \pm 0.6$ \\
\hline $\operatorname{kc} 48(\Delta \operatorname{com} 1)$ & $29.0 \pm 2.1$ & $8.8 \pm 0.9$ \\
\hline $\mathrm{cH} 3035$ ( com 1/COM1) & $24.2 \pm 1.9$ & $9.4 \pm 0.8$ \\
\hline $\operatorname{ckc} 48(\Delta \operatorname{com} 1 / C O M 1)$ & $23.7 \pm 1.6$ & $9.4 \pm 0.7$ \\
\hline
\end{tabular}

a Distance between the tip and basal appendage of the conidia.

${ }^{\mathrm{b}}$ Width of the widest point of the conidium. The mean and standard deviations were calculated based on three independent experiments by measuring at least 100 conidia in each replicate.
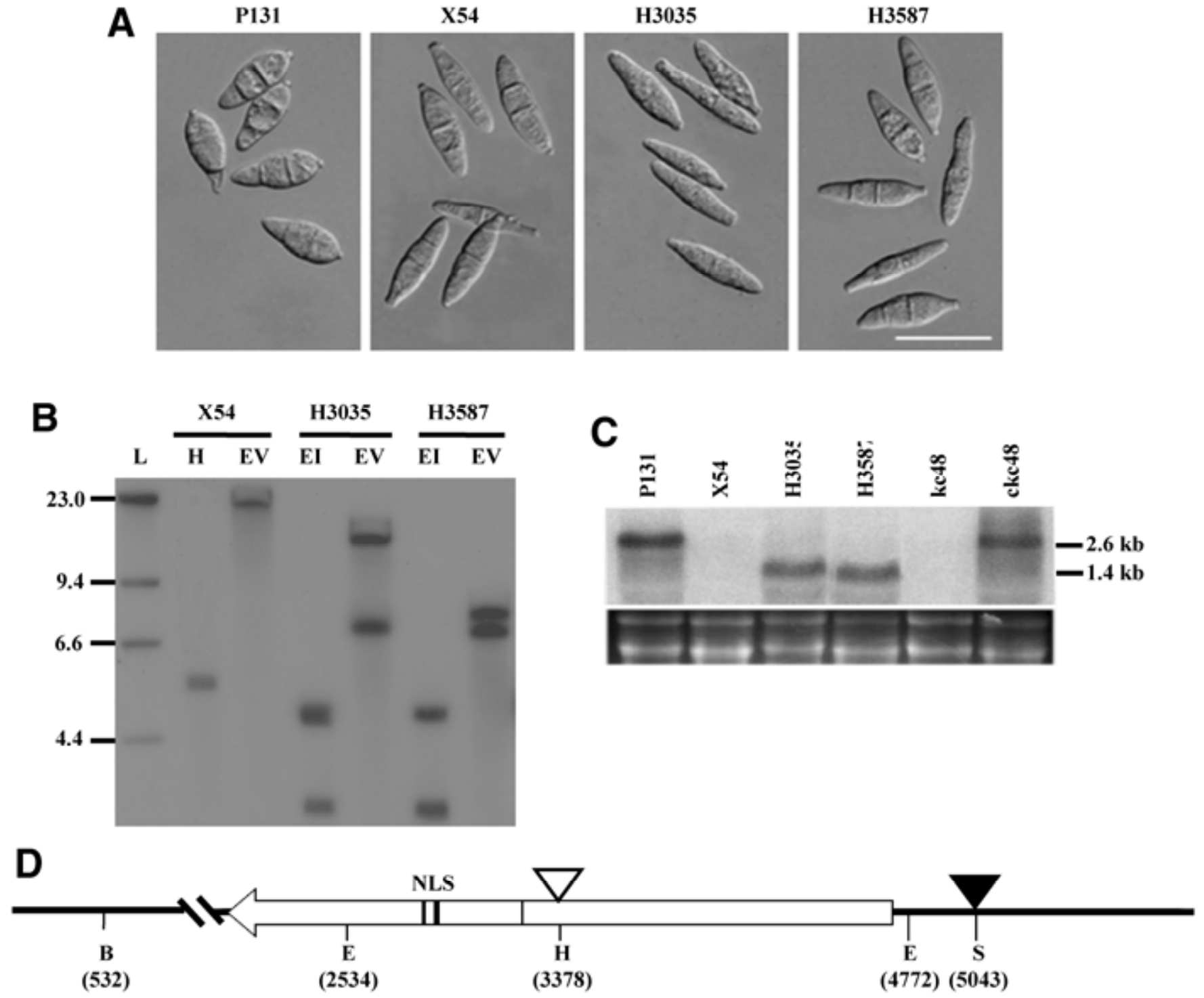

\section{pHEv8 \\ pXB10}

Fig. 1. Wild-type and restriction-enzyme-mediated DNA integration (REMI) mutants with altered conidium morphology. A, Conidia of wild-type strain P131 and REMI mutants X54, H3035, and H3587. Bar $=25 \mu \mathrm{m}$. B, Southern blot of X54, H3035, and H3587 genomic DNA probed with pUCATPH. EI, EcoRI; EV, EcoRV; H, HindIII. L, $\lambda$-HindIII ladder. C, Northern blot analysis with RNA isolated from the wild-type P131; REMI mutants X54, H3035, and H3587; $\Delta$ com 1 mutant kc48; and a complementation strain ckc48 ( $\triangle$ coml/COM1). The lower panel shows that similar amounts of total RNA were loaded in each lane. D, Integration sites of the transforming vector are marked with the black triangle for mutant X54 and the white triangle for mutants H3035 and H3587. Nuclear localization signals (NLS) are marked with black boxes. The horizontal arrow represents the open reading frame of COM1. Plasmids pHEv8 and pXB10 carry the flanking sequences recovered from mutant H3035 and X54, respectively. E, EcoRV; H, HindIII; S, SmaI. 
Genetic crosses were performed between each mutant (MAT1-1) and a wild-type strain S1528 (MAT1-2). Among 52, 31 , and 20 random ascospore progeny isolated from crosses of mutant H3035, H3587, and X54, respectively, all hygromycin susceptible progeny produced normal conidia while all hygromycin-resistant progeny from the X54 cross and most hygromycin-resistant progeny from the $\mathrm{H} 3035$ and $\mathrm{H} 3587$ crosses had conidium morphology defects (data not shown). These data indicated that altered conidium morphology in mutant $\mathrm{H} 3035, \mathrm{H} 3587$, and X54 cosegregated with the hygromycin phosphotransferase (HPT) gene. Because several hygromycinsusceptible progeny from crosses of mutant K2877 and S1005 produced conidia with morphology defects, we concluded that altered conidium morphology in these two mutants did not cosegregate with the HPT gene (data not shown). Therefore, only REMI mutants H3035, H3587, and X54 were selected for further study.

\section{Mutants H3035, H3587, and X54 were disrupted in the COM1 gene.}

Southern blot hybridization probed with the transforming vector pUCATPH (Lu et al. 1994) revealed that mutant X54 had one hybridizing 5.5-kb HindIII fragment and one hybridizing 20-kb EcoRV fragment (Fig. 1B). The same probe hybridized to two EcoRV bands and two EcoRI bands in H3035 and $\mathrm{H} 3587$. However, the 4.8-kb EcoRI band in H3035 appeared to be a doublet (Fig. 1B). These results indicate that the transforming DNA had two integration events in REMI mutants $\mathrm{H} 3035$ and H3587. Although one insertion site must be different, the other integration event occurred in the same genomic region in $\mathrm{H} 3035$ and $\mathrm{H} 3587$.

The plasmid rescue approach was used to isolate the flanking sequences of pUCATPH in X54 and H3035. After selfligation of BamHI-digested X54 DNA, a 4.5-kb fragment of genomic DNA was recovered from plasmid pXB10 (Fig. 1D). Plasmid pHEv8 carrying a $2.2-\mathrm{kb}$ genomic fragment was isolated by self-ligation of EcoRV-digested H3035 DNA. Sequence analysis revealed that the transforming vector was inserted at the $S m a I$ site located 611-bp upstream of the predicted gene MGG_01215.5 (COM1). In H3035, one integration occurred at a HindIII site in the COMI open reading frame (ORF) (Fig. 1D). We also amplified the COM1 allele from $\mathrm{H} 3587$ and found that the transforming vector was inserted into the same HindIII site as in H3035 (Fig. 1D). These data indicated that the REMI mutants H3035, H3587, and X54 were disrupted within the same COM1 gene. On northern blots hybridized with the COM1 ORF, we observed a 2.6-kb band in the wildtype strain P131 but not in mutant X54. In H3035 and H3587, a $1.4-\mathrm{kb}$ band but not the wild-type $2.6-\mathrm{kb}$ band was detected (Fig. 1C), indicating that the insertion events abolished the transcription of COM1 in X54 but only disrupted the synthesis of full-length COM1 transcripts in H3035 and H3587.

To further confirm that disruption of the COM1 gene was responsible for the phenotypes observed in these three REMI mutants, we cloned a $5.5-\mathrm{kb}$ fragment containing the entire COM1 gene into plasmid pKN. The resulting plasmid pKNS2 was linearized by NotI digestion and transformed into the $\Delta$ coml mutant. All 14, 15, and 10 neomycin-resistant transformants of $\mathrm{H} 3035, \mathrm{H} 3587$, and $\mathrm{X} 54$, respectively, isolated in this study produced conidia with normal morphology (Table 1). These data indicated that the COM1 gene complemented the conidium morphology defects of these REMI mutants.

The COMI cDNA was amplified by reverse-transcription polymerase chain reaction (PCR) from RNA isolated from P131 conidia. The transcription initiation and termination sites were determined by $5^{\prime}$ and $3^{\prime}$ rapid amplification of cDNA ends reactions. The resulting data indicated that the full-length
COM1 transcript was 2,591 bp, including a 2,325-bp ORF plus a 100-bp 5' and 166-bp 3' untranslated region. Two introns in the COM1 ORF region were identified. Although the first intron (nucleotides 172 to 437) was predicted correctly by the Broad Institute, the second intron should be from 702 to 772 instead of from 774 to 878 . BLASTP search against GenBank indicated that Com 1 is similar only to hypothetical proteins from other filamentous ascomycetes. Secondary structure analysis by SSpro revealed that the Com 1 protein contains a predicted loop consisting of random coils and two flanking $\alpha$-helix modules (amino acid residues 213 to 337 and 680 to 740; Supplementary Fig. 1), which are similar to the helix-loop-helix structure characteristic of many transcription factors (Massari and Murre 2000). Interestingly, the COM1 orthologs from filamentous ascomycetes were more conserved in the putative $\alpha$ helix regions than in the coil modules.

\section{The COM1 gene replacement mutants are reduced in virulence.}

To further characterize the function of $C O M 1$, the gene replacement vector pCOM1-2.4 was constructed by replacing the COM1 ORF with the HPT gene (Fig. 2A). The neomycin resistance gene $N P T I I^{\mathrm{R}}$ was carried on the vector backbone outside the gene replacement cassette. After NotI digestion, pCOM1-2.4 was transformed into the wild-type strain P131. A total of 45 hygromycin-resistant, neomycin-sensitive transformants were isolated and screened by PCR with the primer pairs P1/P2, P3/P4, and P5/P6 (Fig. 2A). Four com1 deletion mutants were identified and confirmed by Southern blot analysis (Fig. 2B). When probed with a 1.1-kb fragment amplified from pCOM1-2.4 with the primers Lf and Lr, the $\Delta$ coml mutants kc2, kc4, kc9, and kc48 had a 4.7-kb KpnI band instead of the wild-type 3.7-kb band (Fig. 2B).

Colonies formed by the $\Delta$ coml mutants on oatmeal tomato agar (OTA) plates had normal growth rates and a typical grayish appearance (data not shown). However, the $\Delta$ com 1 mutants had conidium morphology defects similar to those of the original com1 REMI disruption mutants (Fig. 2C; Table 1). Conidia produced by the $\Delta \mathrm{com} 1$ mutants had longer basal and apical cells and narrower middle cells than those of the wild-type conidia (Table 1). The overall length of $\Delta$ coml conidia $(29.1 \pm$ $2.1 \mu \mathrm{m})$ was significantly longer than those of P131 conidia $(23.4 \pm 1.9 \mu \mathrm{m})$. In addition, conidiation was reduced over four times in the $\Delta$ coml mutants (data not shown). These data indicated that the COM1 gene is required for normal asexual reproduction but not for vegetative growth.

In infection assays, a few typical blast lesions developed on rice leaves sprayed with the coml REMI and deletion mutants (Fig. 3A). However, the number of lesions appearing on leaves sprayed with kc48 and $\mathrm{H} 3035$ was significantly less than that of the wild-type strain P131 (Fig. 3A; Table 2). The complemented transformants ckc48 and $\mathrm{cH} 3035$ expressing a copy of the wild-type COM1 ectopically were as virulent as P131 (Table 2). Similar results were observed in infection assays with barley leaves (Fig. 3B). We also noticed that lesions caused by the $\Delta$ coml mutants tended to be smaller than those of P131 (Fig. $3 \mathrm{~A})$. The vast majority of the small necrotic spots caused by the $\Delta$ coml mutants failed to develop into full-size, normal blast lesions even after prolonged incubation up to 10 days (data not shown). On barley leaves, typical lesions with extensive necrotic regions surrounding the green islands were rarely observed (Fig. $3 \mathrm{~B})$. These data indicated that the COMI gene is not essential for pathogenesis but is required for full virulence.

\section{The $\Delta$ com1 mutants are defective in penetration.}

In penetration assays with onion epidermal cells, conidia produced by the $\Delta$ coml mutants were normal in germination 
and appressorium formation. However, appressorial penetration was reduced significantly in the $\Delta$ coml mutants. By $24 \mathrm{~h}$, $82.0 \pm 5.7 \%$ of the wild-type appressoria penetrated and developed infectious hyphae. Under the same conditions, only 40.0 $\pm 4.5 \%$ of the appressoria formed by the $\Delta$ coml mutant kc48 penetrated the onion epidermal cells (Fig. 4A). Similar defects in appressorial penetration were observed in the original REMI mutants $\mathrm{H} 3035$ and $\mathrm{H} 3587$ (data not shown).

The $\Delta$ coml mutants also differed from the wild-type strain in the extent of infectious growth and morphology of infectious hyphae. By $36 \mathrm{~h}$, infectious hyphae formed by P131 in the onion epidermal cells had 3 to 10 branches on average. In contrast, the $\Delta$ com 1 mutants usually had only 1 to 3 branches (Fig. 4B). In penetration assays with rice leaf sheath epidermal cells, similar defects in penetration and growth of infectious hyphae were observed in the $\Delta$ coml mutants (data not shown). These data indicated that the COM1 gene plays an important role in appressorial penetration and in planta growth of infectious hyphae.

\section{Deletion of $C O M 1$ adversely affected appressorium turgor generation.}

Because the $\Delta$ coml mutant was defective in appressorial penetration, we assayed turgor pressure in appressoria. When treated with 25\% PEG-8000 (polyethylene glycol molecular weight 8,000; Sigma, St. Louis), less than $5.0 \%$ of appressoria underwent cytorrhysis in the $\Delta$ coml mutant kc48 and the wildtype strain P131 (Fig. 5A). When treated with 30\% PEG-8000, over $82.0 \%$ of the appressoria of the $\Delta \mathrm{coml}$ mutant collapsed but less than $25 \%$ of the wild-type plasmolyzed. Significant
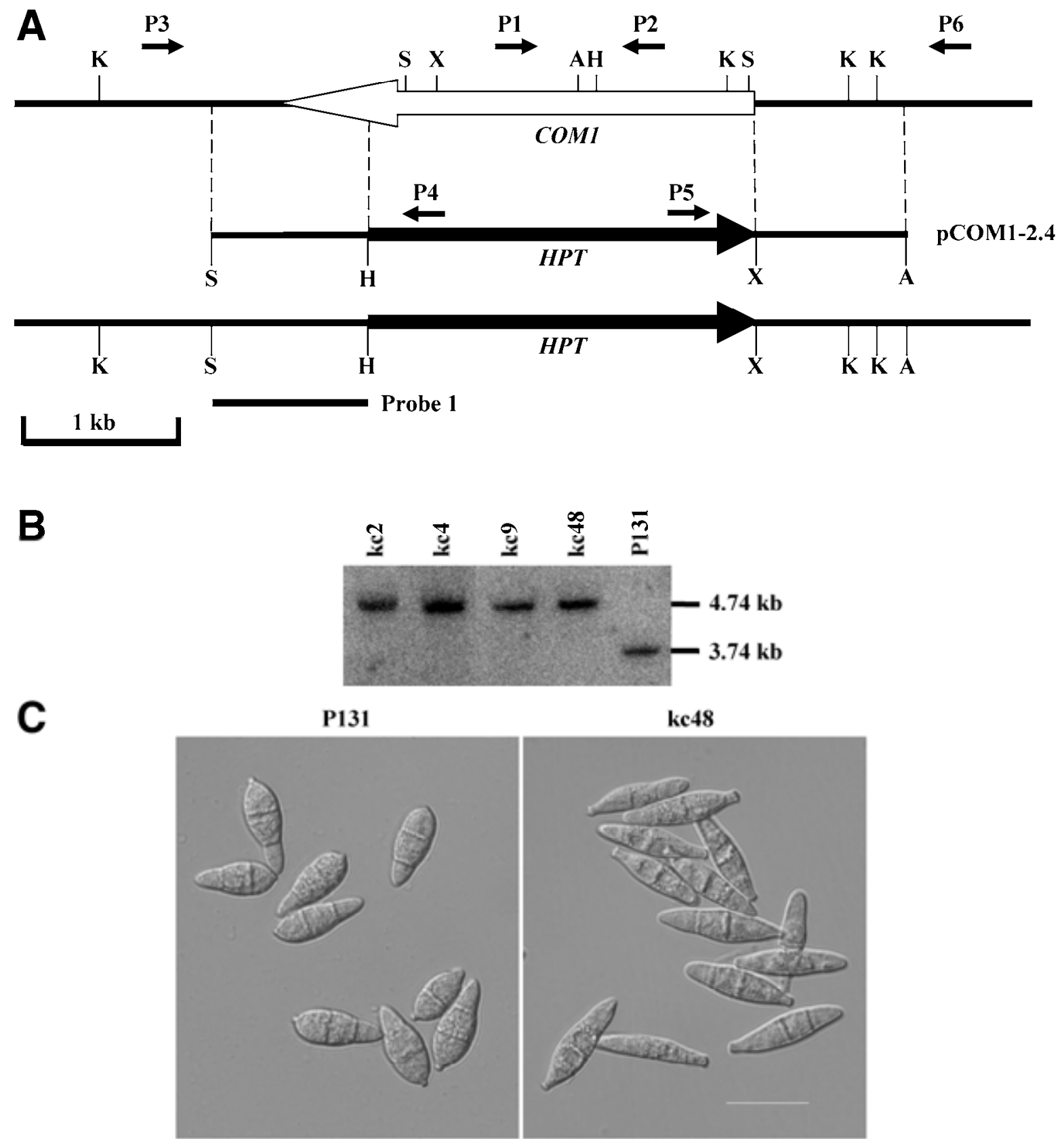

Fig. 2. Targeted deletion of the COM1 gene. A, COM1 gene and gene replacement vector pCOM1-2.4. The gene replacement vector was constructed by replacing the majority of COM1 with the hygromycin phosphotransferase gene $(H P T)$. The positions of primers P1, P2, P3, P4, P5, and P6 are indicated with small arrows. A, ApaI; H, HindIII; K, KpnI; S, SpeI; X, XhoI. B, Southern blot of KpnI-digested genomic DNAs of $\Delta c o m 1$ mutants kc2, kc4, kc9, kc48, and wild-type P131 hybridized with Probe 1 . C, Conidia of the wild-type P131 and the $\Delta$ com 1 mutant $\mathrm{kc} 48$. Bar $=20 \mu \mathrm{m}$. 
differences in the percentage of appressoria undergoing cytorrhysis were also observed between the wild-type and $\Delta$ coml mutant strains in the presence of 35\% PEG-8000 (Fig. 5A). These data indicated that the $\Delta \mathrm{coml}$ mutant was defective in appressorium turgor generation, which may be responsible for the reduction in penetration efficiency.

Conidia from the wild-type and $\Delta$ coml mutant strains also were stained with osmium tetroxide and examined under transmission electron microscopy (TEM) for changes in subcellular structures (Kang et al. 2005). In longitudinal sections, numerous lipid body-like droplets (LBD) were observed in the middle and basal compartments of the P131 conidia (Fig. 5B and D). These LBD tended to aggregate and distribute along the edges of the conidial cells, which was likely due to the central vacuoles. The $\Delta$ coml mutant kc48 had considerably fewer LBD than the wild-type conidia, particularly in the middle and basal compartments (Fig. 5C and E). LBD in the conidia of the $\Delta$ coml mutant also were smaller than those of P131 and normally did not form massive aggregates along the cell wall (Fig. $5 \mathrm{~B}$ to $\mathrm{E}$ ), indicating that $C O M 1$ was involved in the formation and distribution LBD in conidia. Because lipid bodies of conidium cells play an important role in glycerol accumulation in the appressoria (Howard et al. 1991; de Jong et al. 1997), the reduction in lipid bodies may be responsible for or contribute to the defects of the $\Delta \mathrm{com} 1$ mutant in generation of normal appressorium turgor.

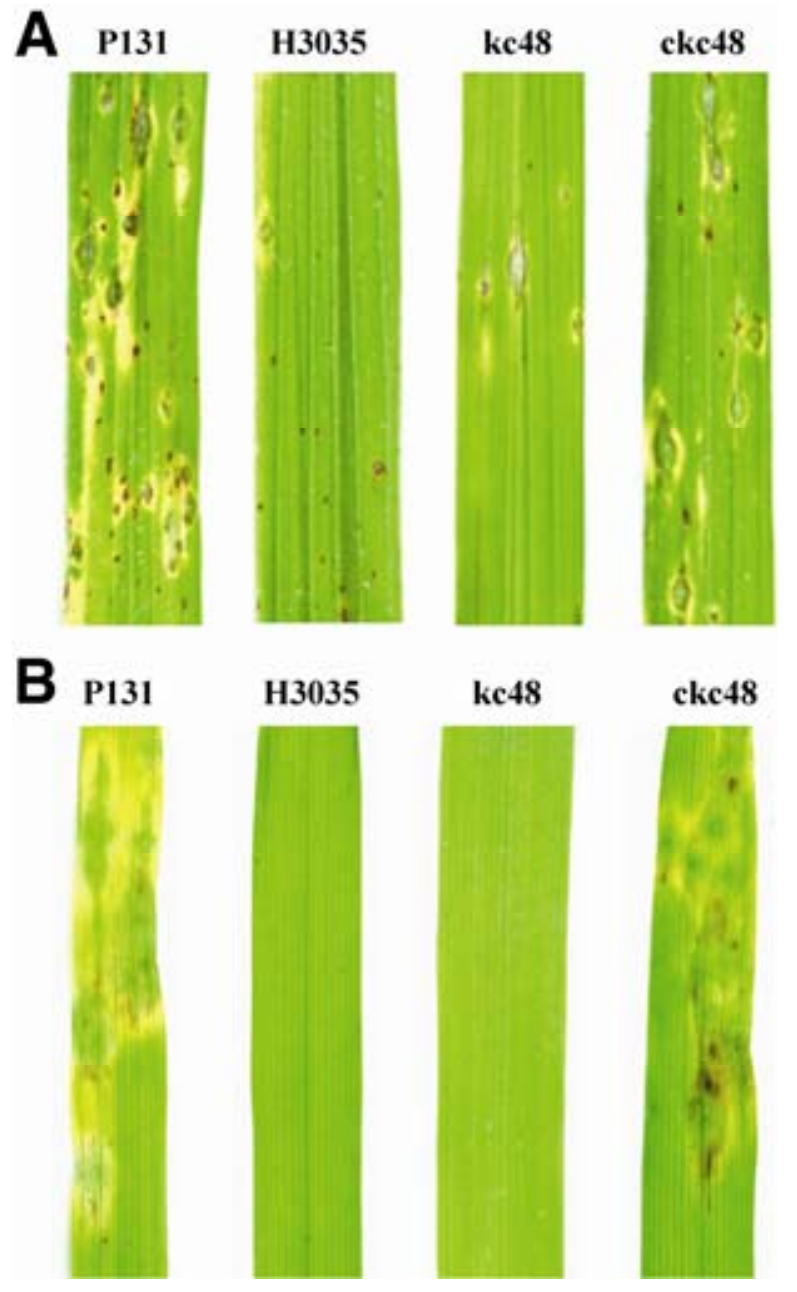

Fig. 3. Plant infection assays. A, Rice and $\mathbf{B}$, barley seedlings were sprayed with the wild-type P131, restriction-enzyme-mediated DNA integration mutant $\mathrm{H} 3035$, $\Delta$ com 1 mutant $\mathrm{kc} 48$, and a complemented transformant ckc48. Typical leaves were photographed 5 days after inoculation.

\section{Defects in morphology affected the sedimentation rate of $\Delta$ com 1 conidia.}

To assay whether changes in conidium morphology had any effect on conidium dispersal, we conducted sedimentation tests with the $\Delta$ coml mutant using a water-filled $80-\mathrm{cm}$ column with a constant elution speed. For the wild-type strain, conidia became detectable in the 4th elution tube and peaked from the 16 th to 18 th tubes (Fig. 6). Conidia of the $\Delta$ coml mutant were reduced in sedimentation speed. The 10th tube was the first tube with detectable amounts of conidia. The 33rd to 35th tubes had high concentrations of conidia. In general, the elution tubes collected after the 24th tube had more conidia in the $\Delta$ coml mutant than in the wild-type isolate, indicating that the conidia of the $\Delta c o m 1$ mutant had a reduced sedimentation rate.

\section{The expression pattern of $C O M 1$.}

To determine the expression pattern and promoter activity of COM1, a $1.0-\mathrm{kb}$ fragment of the COM1 promoter was amplified and cloned into the vector pKNTG. The resulting plasmid pECOM1 containing the $\mathrm{P}_{\mathrm{COM} 1}$-enhanced green fluorescent protein (eGFP) construct was used to transform the wild-type strain P131. Two pECOM1 transformants were identified by PCR and confirmed by Southern blot analysis (data not shown). In these two $\mathrm{P}_{\mathrm{COM} 1}$-eGFP transformants, no obvious changes in growth or virulence were observed (data not shown). eGFP signals were present in the cytoplasm of vegetative hyphae, conidia, appressoria, and infection hyphae (Fig. 7). These data suggested that the COM1 promoter may be constitutively active in $M$. oryzae. By searching the $M$. oryzae expressed sequence tag databases, we found that COM1 transcripts were present in cDNA libraries constructed with RNA isolated from the mycelium, germinated conidia, appressoria, and mating cultures (Soanes and Talbot 2005).

\section{Expression and subcellular localization of Com1-eGFP fusion proteins.}

We generated a COM1-eGFP fusion construct pKG31 that is under the control of its native promoter (Fig. 8A). After transforming this construct into the $\Delta \mathrm{coml}$ mutant kc48, 20 transformants, in total, were isolated and all of these produced normal conidia. Unfortunately, none of the transformants had detectable eGFP signals in vegetative hyphae, conidia, germination tubes, appressoria, or infection hyphae (Fig. 8B). To determine whether the COM1 C-terminal fusion might interfere with detection of eGFP, we also generated an eGFP-COMI fusion construct pKG32 (data not shown) and transformed it into kc48. All 19 resulting transformants produced normal conidia but eGFP signals were not detectable at various developmental and infection stages (data not shown). These results indicated that fusion of $e G F P$ to either the $\mathrm{N}$ or $\mathrm{C}$ terminus of $C O M 1$ had no effect on its function. However, the accumulation of the eGFP fusion proteins may be too low to be detected by fluorescence microscopy in these transformants. This could be

Table 2. Rice infection assays

\begin{tabular}{|c|c|c|}
\hline Strains & Typical blast lesions ${ }^{\mathrm{a}}$ & Small necrotic spots ${ }^{b}$ \\
\hline P131 (wild-type) & $73.2 \pm 28.9$ & $120.6 \pm 72.8$ \\
\hline $\mathrm{H} 3035$ (com 1$)$ & $2.4 \pm 3.7$ & $62.6 \pm 39.2$ \\
\hline $\operatorname{kc} 48(\Delta \operatorname{com} 1)$ & $3.6 \pm 3.6$ & $89.8 \pm 48.4$ \\
\hline $\mathrm{cH} 3035$ (com $1 /$ COM1) & $54.0 \pm 19.7$ & $75.0 \pm 24.9$ \\
\hline $\operatorname{ckc} 48(\Delta \operatorname{com} 1 / C O M 1)$ & $45.6 \pm 10.6$ & $55.2 \pm 19.4$ \\
\hline
\end{tabular}

${ }^{\mathrm{a}}$ Lesions with gray centers and larger than $2 \mathrm{~mm}$ in diameter.

${ }^{b}$ Brown spots without gray centers and smaller than $2 \mathrm{~mm}$ in diameter. Means and standard deviations were calculated from the lesions formed on the middle $5 \mathrm{~cm}$ of the fourth leaf. Three independent experiments were conducted with at least 10 leaves in each replicate. 
A

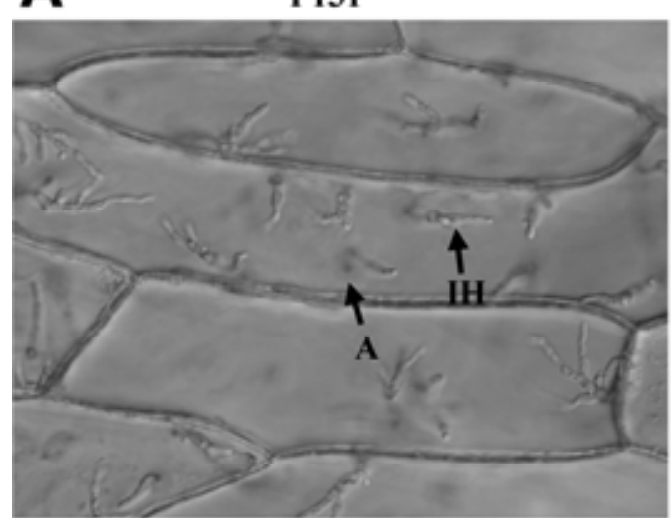

\section{B}

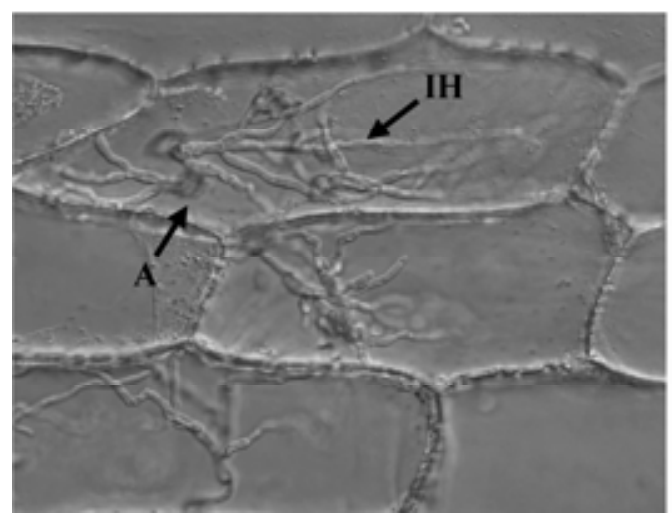

kc48

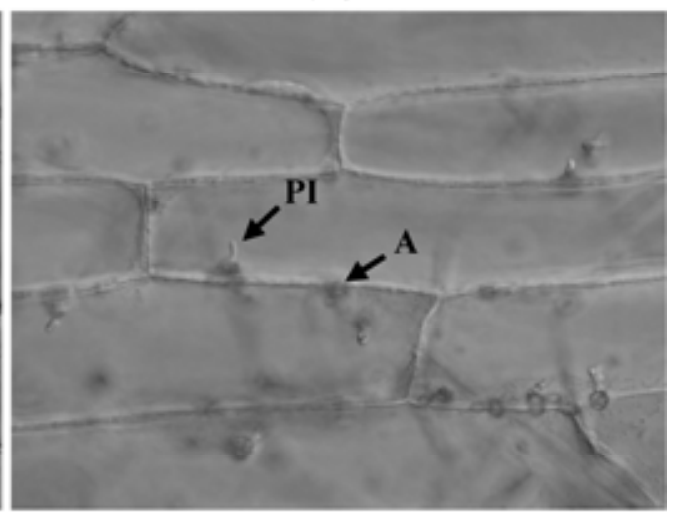

kc48

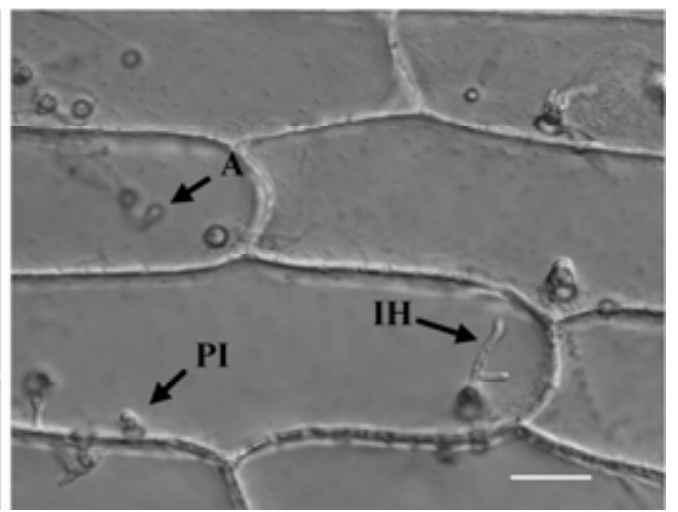

Fig. 4. Appressorial penetration assays. Onion epidermal cells were inoculated with conidia of the wild-type P131 and $\Delta$ coml mutant kc48. Penetration and infectious hyphal growth were assayed at $\mathbf{A}, 24 \mathrm{~h}$ and $\mathbf{B}, 36 \mathrm{~h}$ after inoculation. Bar $=20 \mu \mathrm{m}$. A, appressoria; IH, infectious hyphae; PI, primary infectious hyphae.
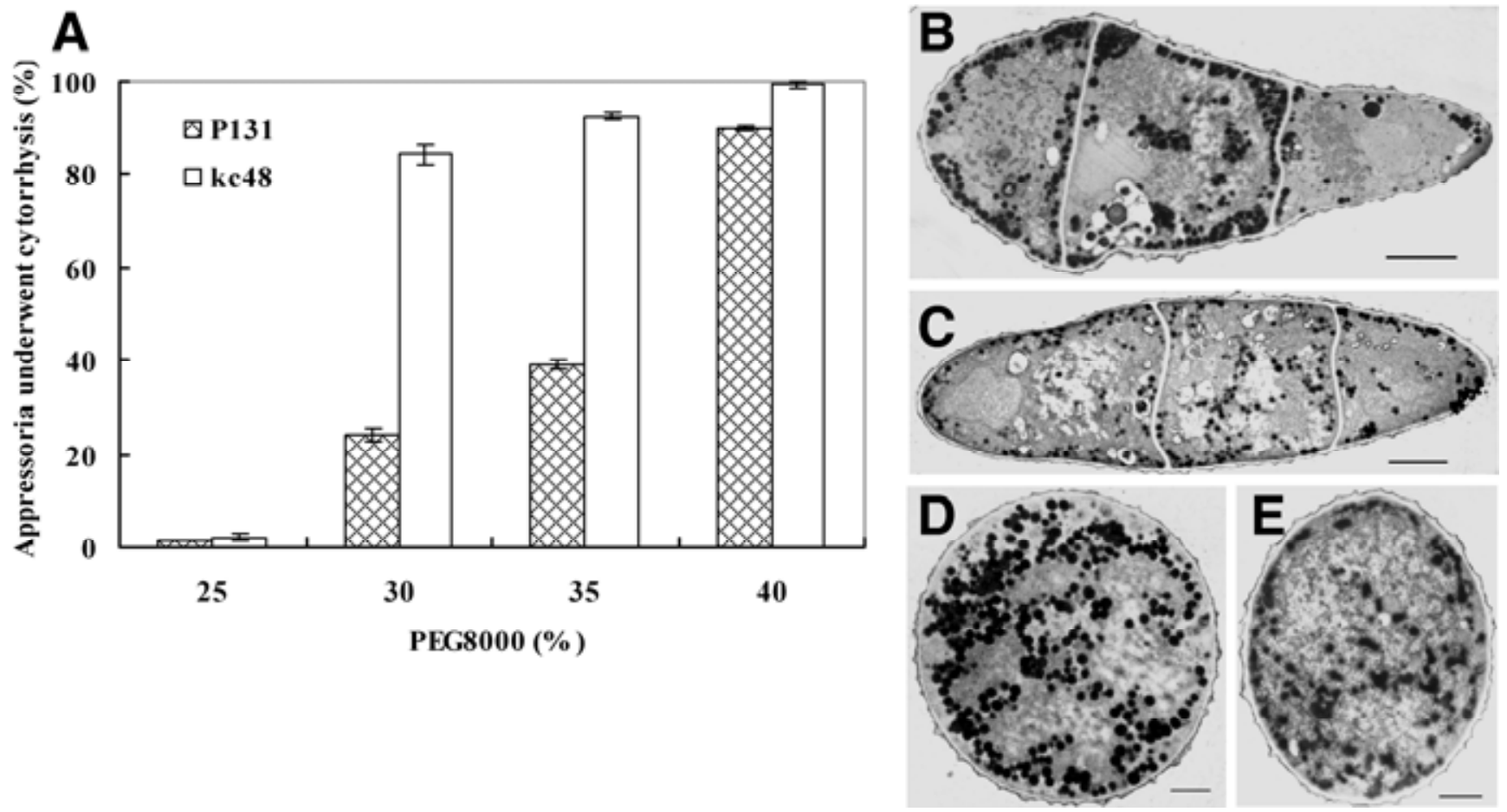

Fig. 5. Defects of the $\Delta$ com 1 mutant in turgor generation. A, Cytorrhysis assays to assess appressorium turgor. The percentages of appressoria that underwent cytorrhysis were recorded with 25, 30, 35, and 40\% PEG-8000 (polyethylene glycol, molecular weight 8,000) (wt/vol). At least 300 mature appressoria formed by the wild-type P131 and $\Delta$ com1 mutant kc48 strains were examined for each experiment. Mean and standard errors were calculated from three independent replicates. B through $\mathbf{E}$, Transmission electron microscopy of conidia from the wild-type and $\Delta$ coml mutant stains. B and C, Longitudinal sections and $\mathbf{D}$ and $\mathbf{E}$, cross-section views of representative conidia of P131 and kc48, respectively. The sections were stained with osmium tetroxide. $\mathrm{Bar}=3 \mu \mathrm{m}(\mathrm{B}$ and $\mathrm{C})$ and $1 \mu \mathrm{m}(\mathrm{D}$ and $\mathrm{E})$. 
caused by either the expression level of the COM1-eGFP construct or the stability of Com1-eGFP fusion proteins. Fusion of the full-length COMI gene may have an adverse effect on the proper folding of eGFP.

Because the Com1 protein contained three putative nuclear localization signal (NLS) sequences at its C-terminus region, we also generated the $C O M 1^{335-613}$-eGFP fusion construct (pDWCOM1-2) under the control of the RP27 promoter (Bourett et al. 2002) and transformed it into the wild-type

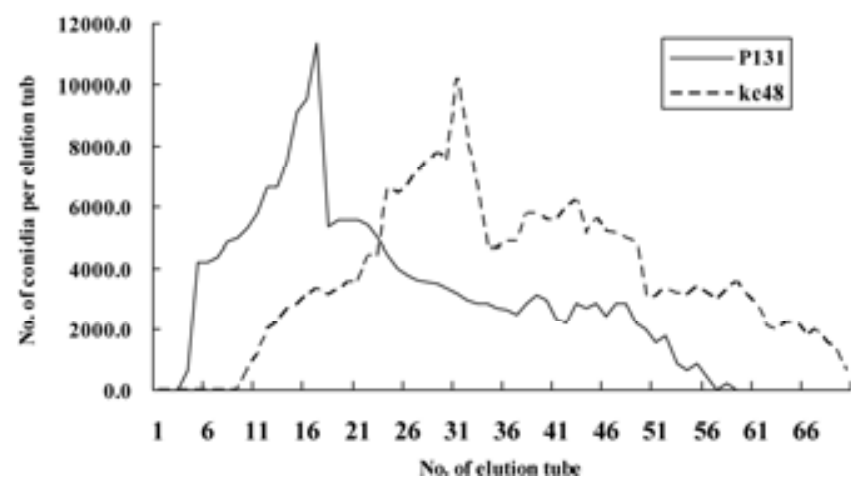

Fig. 6. Sedimentation rates of conidia harvested from the wild-type P131 and $\Delta$ com 1 mutant $\mathrm{kc} 48$. The number of conidia in each elution tube (numbered from 1 to 70 ) was counted and plotted.
P131. The resulting transformants had strong fluorescent signals in the nuclei of conidia (Fig. 8C). Nuclear localization of $C O M 1^{335-613}$-eGFP fusion proteins also was observed in vegetative hyphae, appressoria, and infectious hyphae formed inside onion epidermal cells (Fig. 8C). These results suggested that fusion of the C-terminal portion of COM1 with eGFP, unlike fusion with the full-length gene, had no effect on green fluorescence and led to nuclear location of the fusion proteins.

The PPVKRPRE and PLAKKFK sequences can direct nuclear localization of Com ${ }^{335-613}$-eGFP fusion proteins.

To determine their role in the nuclear localization of Com $1^{335-613}$-eGFP, we deleted three predicted NLS sequences individually in pDWCOM1-2 and transformed the NLS deletion constructs into the wild-type P131. In transformants expressing the construct pDWCOM1-21 in which the first NLS KPKK sequence was deleted from the $C O M 1^{335-613}$-eGFP construct, strong fluorescent signals were observed in the nucleus (Fig. 9). In contrast, in transformants expressing the $C O M 1^{\triangle \mathrm{PPVKRPRE}}$ - and

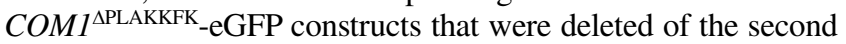
and third putative NLS sequences in $C O M 1^{335-613}$, respectively, fluorescent signals were observed in the cytoplasm of conidium cells (Fig. 9). These results suggested that both the PPVKRPRE and PLAKKFK sequences may be important for nuclear localization of the Com $1^{335-613}$-eGFP fusion protein. The PPVKRPRE sequence appeared to be essential because fluorescent signals were observed only in the cytoplasm in the $C O M 1^{\triangle P \text { PVKRPRE }}$

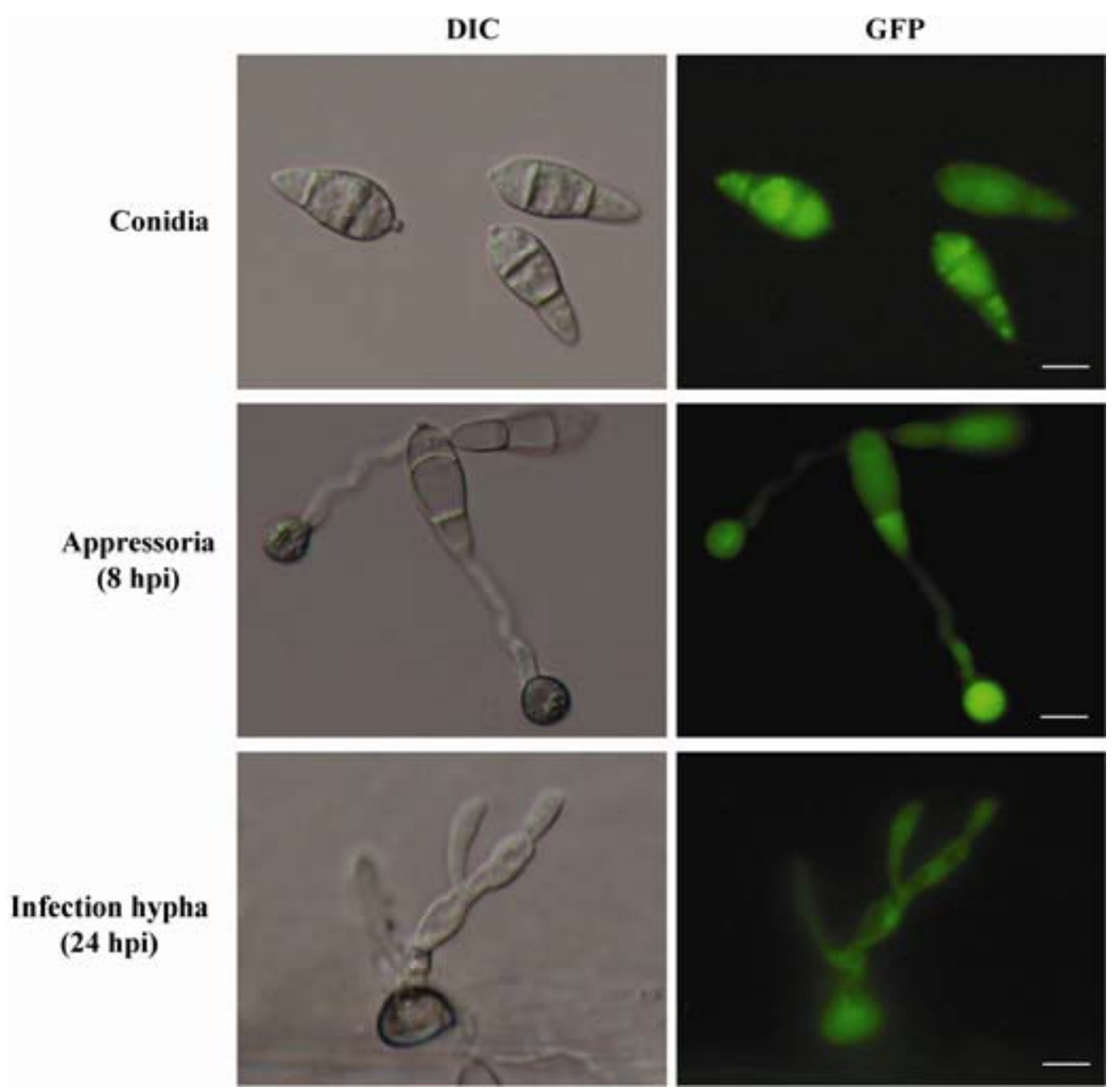

Fig. 7. Constitutive expression of COM1. Fresh conidia, appressoria ( $8 \mathrm{~h}$ postinoculation [hpi]), and infectious hyphae (24 hpi) of a transformant of P131 expressing the pECOM1 construct (eGFP [enhanced green fluorescent protein gene) controlled by the COM1 promoter) were examined under differential interference contrast (DIC) and epifluorescence (GFP) microscopy. Bar $=10 \mu \mathrm{m}$. 
eGFP transformants. In transformants expressing the $C O M 1^{\triangle P{ }^{2} A K K F K}-$ eGFP construct, a few nuclei still had stronger fluorescent signals than cytoplasm (Fig. 9).

The PPVKRPRE sequence was dispensable for the biological function of Com1.

Because the PPVKRPRE sequence was essential for nuclear localization of Com $1^{335-613}$-eGFP, we also deleted it from the full-length COM1-eGFP fusion construct pKG31. The resulting plasmid, pKG31-NLS2, was transformed into the $\Delta$ coml mutant kc48. Fifteen transformants were generated and confirmed by PCR analysis to contain the $C O M 1^{\triangle \text { PPVKRPRE}_{-} \mathrm{eGFP}}$ construct. Similar to the transformants expressing the fulllength $C O M 1$-eGFP fusion, none of these $C O M 1^{\triangle P P V K R P R E}$ eGFP transformants had detectable eGFP signals in hyphae, conidia, or appressoria (data not shown). Surprisingly, all these

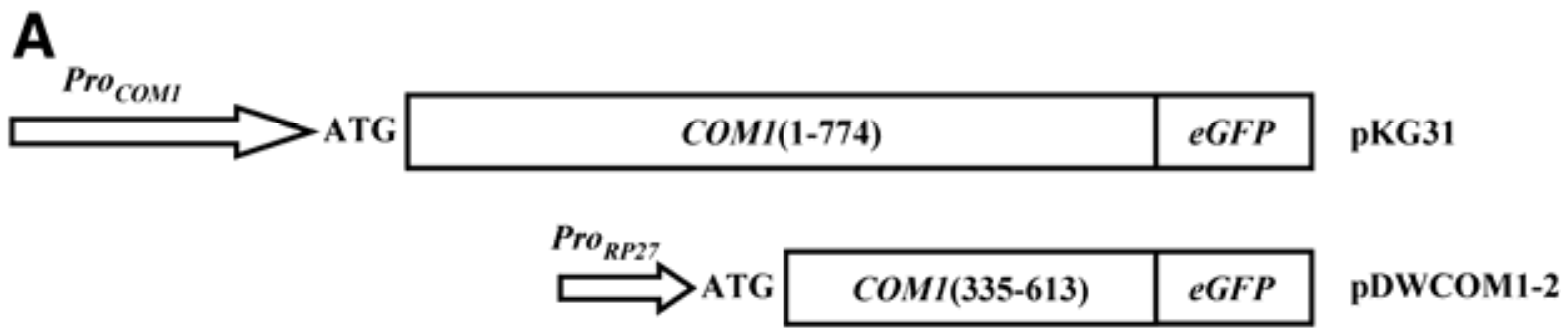

B

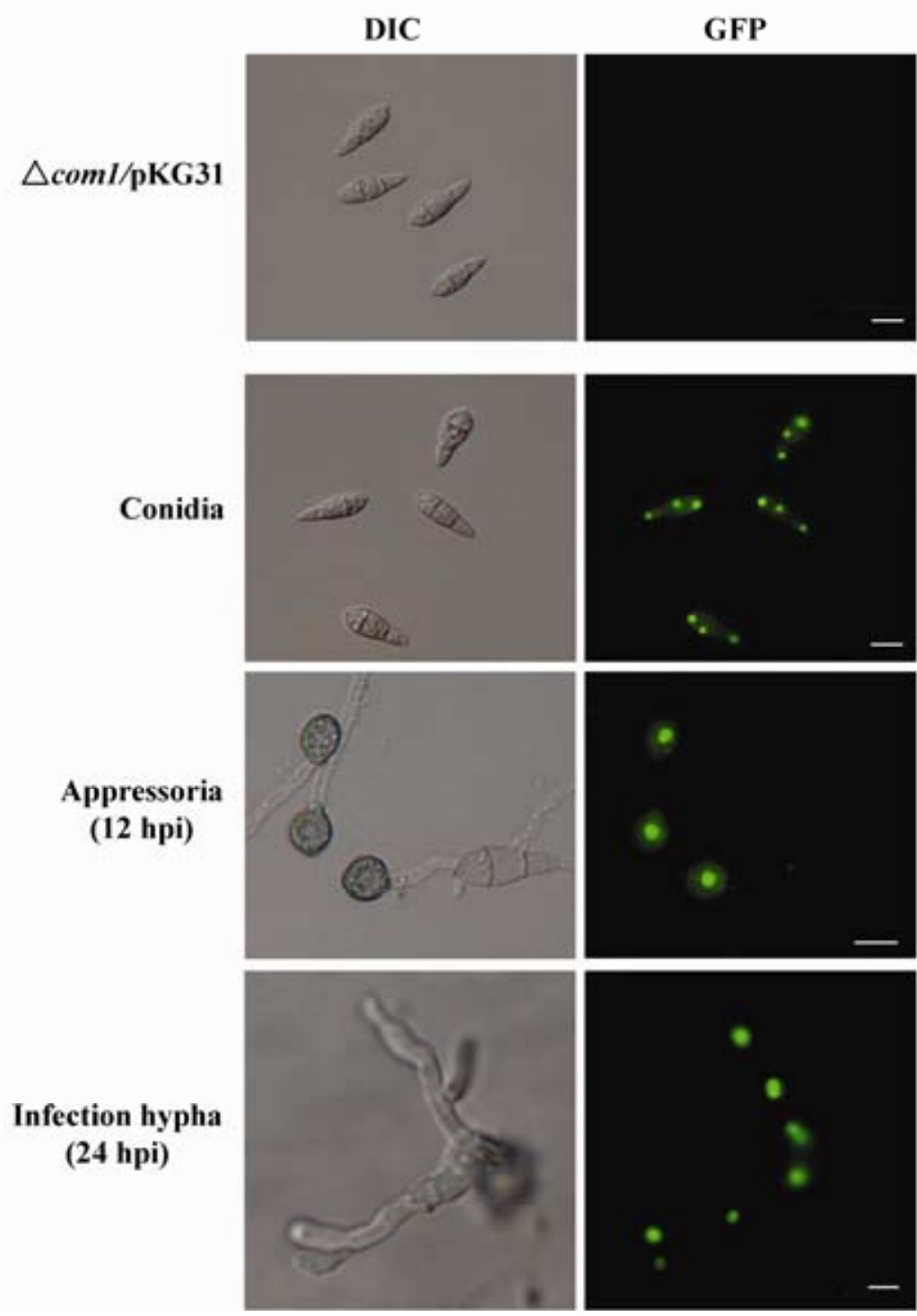

Fig. 8. Localization of Com1-eGFP (enhanced green fluorescent protein) fusion proteins. A, Diagrams of two eGFP fusion constructs. The full-length COM1 open reading frame fused with $e G F P$ was under the control of the COM1 native promoter in pKG31. The partial COM1 ${ }^{335-613}-e G F P$ fusion construct pDWCOM1-2 was under the control of the RP27 promoter. B, No eGFP signal detectable in the conidia of transformant expressing COM1-eGFP (pKG31). C, Nuclear localization of eGFP signals observed in the conidia, appressoria (12 h postinoculation [hpi]), and infectious hyphae ( $24 \mathrm{hpi})$ of a transformant of the wild-type strain expressing the pDWCOM1-2 construct. All the observations were made under differential interference contrast (DIC) and epifluorescence (GFP) microscopy. Bar $=10 \mu \mathrm{m}$. 
transformants produced normal conidia (Supplementary Fig. 2) and were as virulent as the wild-type strain in rice infection assays (data not shown), indicating that the COMI $1^{\triangle \mathrm{PPVKRPRE}}$ eGFP allele fully complemented the $\Delta$ com 1 mutant. Therefore, this PPVKRPRE sequence was not essential for COM1 function although it could direct the nuclear localization of Com $1^{335-613}$-eGFP fusion proteins.

\section{DISCUSSION}

Over the past decade, M. oryzae has emerged as a model to study fungus-plant interactions and infection-related developmental processes in fungal pathogens. Large-scale insertional mutagenesis has been used to identify mutants that are defective in growth and infection (Guptaa and Chattoo 2007, 2008; Jeon et al. 2008). However, we believe that conidium development and morphogenesis is underinvestigated even though conidia play a critical role in the infection cycle of $M$. oryzae. In this study, we focused on one class of mutants identified by REMI mutagenesis that produced slender conidia. In three of the mutants with abnormal conidium morphology, the transforming vector was integrated into the COM1 gene. The protein encoded by COM1 has a putative helix-loop-helix structure that is characteristic of many transcription factors (Massari and Murre 2000). The COMI homologs are well conserved in other filamentous ascomycetes, including Chaetomium globosum, Neurospora crassa, Fusarium graminearum, A. fumigatus, and A. nidulans. However, none of these COM1 homologs has been functionally characterized. Basidiomycetes and ascomycetous yeasts belonging to orders Saccharomycetales or Archiascomycetes lack COM1 homologs, suggesting that COM1 is unique to filamentous ascomycetes.

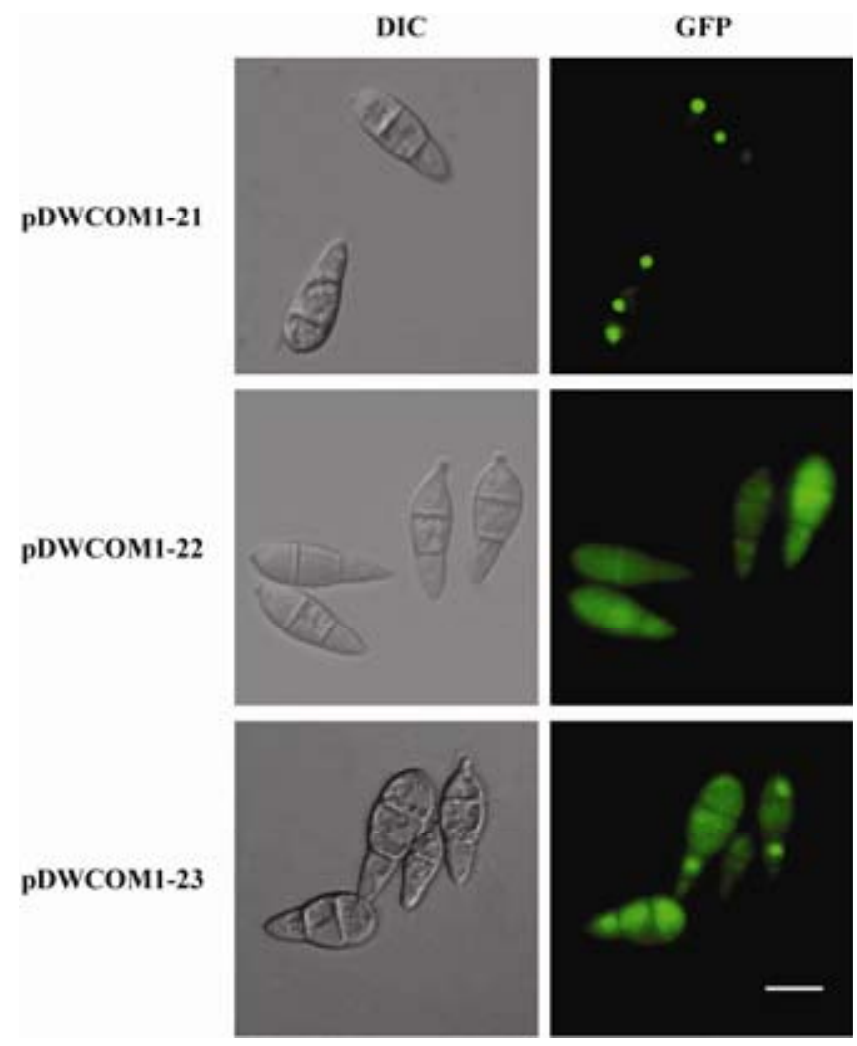

Fig. 9. Functional characterization of three predicted nuclear localization signal sequences in COM1. Transformants expressing pDWCOM1-21

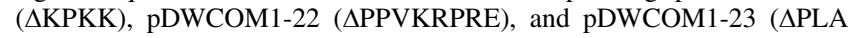
KKFK). All the observations were made under differential interference contrast (DIC) and epifluorescence (GFP) microscopy. Bar $=10 \mu \mathrm{m}$.
Conidia produced by the $\Delta \mathrm{com} 1$ mutants were $25 \%$ longer and had narrower basal and middle cells than those of the wild-type strain. It is likely that COM1 is involved in regulating conidial development and normal conidium morphology in $M$. oryzae. In addition to conidium morphology defects, the coml disruption and deletion mutants had pleiotropic defects in appressorial penetration, infectious growth, and virulence (Figs. 3 and 4; Table 2). Cytorrhysis assays with mature appressoria indicated that the $\Delta$ coml mutant was defective in the generation of normal appressorium turgor (Fig. $5 \mathrm{~A}$ ), which played a critical role in appressorial penetration. In $M$. oryzae, appressorium turgor is generated by the accumulation of a high concentration of intracellular glycerol. Lipid bodies, glycogens, and trehalase are major carbon storage products in conidium cells that contribute to glycerol biosynthesis in appressoria (de Jong et al. 1997; Talbot 2003). TEM examinations showed that conidia of the $\Delta$ com 1 mutant produced fewer, smaller, and less aggregated LBD than those of the wild-type fungal isolate (Fig. 5). Therefore, COM1 may be involved in regulating turgor generation in appressoria.

When analyzed with the PSORTII and PredictNLS programs, Com1 protein was predicted to be localized to the nuclei. However, although the COM1-eGFP or eGFP-COM1 fusion constructs under the control of its native promoter complemented the $\Delta$ com 1 mutant, no eGFP signals in the nucleus or cytoplasm were observed in different developmental and infection stages. Because fluorescence signals were easily detectable in transformants expressing the pECOM1 construct in which eGFP was under the control of the same COMI promoter (Fig. 8), the promoter activity of this $1.0-\mathrm{kb}$ upstream fragment should be sufficient to constitutively express these fusion constructs. Therefore, although it remains possible that the expression level of the COMI-eGFP and eGFP-COMI construct was too low to be detected by epifluorescence microscopy, most likely fusion of the full-length COM1 gene may have an adverse effect on the folding and fluorescence of the eGFP fusion protein.

Interestingly, when the C-terminal portion of COM1 (residue 335 to 613) containing three predicted NLS sequences was fused to eGFP and expressed in the wild-type strain with the RP27 promoter, eGFP signals were observed in the nuclei of the conidia, vegetative hyphae, germ tubes, appressoria, and infectious hyphae. The difference between transformants expressing eGFP fused with the full-length or C-terminal portion of COMI gene could be related to different promoters that were used to express these fusion constructs. However, because eGFP signals were easily detected in the cytoplasm of $\mathrm{P}_{\mathrm{COM} 1-\mathrm{eGFP}}$ transformants, it is more likely that only fusion of the C-terminal portion of COMI but not the full-length gene with eGFP could produce a functional fluorescent protein with normal folding.

Because Com1 $1^{335-613}$-eGFP localized to the nucleus, we examined the function of three predicted NLS sequences by deletion analyses. Although the NLS KPKK sequence was dispensable, both the PPVKRPRE and PLAKKFK sequences appeared to be important for nuclear localization of Com $1^{335-613}$ eGFP (Fig. 9). Because the PPVKRPRE was essential for directing the localization of the COM1 $1^{\triangle 335-613}$-eGFP fusion protein to nuclei (Fig. 9), we also tested its role in the function and subcellular localization of the full-length COM1 gene. To our surprise, deletion of the PPVKRPRE sequence had no obvious effect on the biological function of Com 1 because the $C O M 1^{\triangle \mathrm{PPVKRPRE}}$ allele fully complemented the $\Delta$ coml mutant. Therefore, the PPVKRPRE sequence is unlikely to be important for subcellular localization of Com1, although it can function as an NLS in the Com $1^{335-613}$-eGFP fusion protein. There- 
fore, protein folding and localization may be different between the Com1-eGFP and Com $1^{335-613}$-eGFP fusion proteins. It will be important to determine the function of the PLAKKFK sequence, a putative NLS of Com1, which also was important for nuclear localization of Com $1^{335-613}$-eGFP fusion proteins.

In comparison with other known conidium morphology mutants, the phenotype of the coml deletion mutant appeared to be unique. The con 1 mutant produced elongated conidia similar to those of the coml mutant. However, conl conidia were defective in appressorium formation (Shi and Leung 1995). The con 2 mutant produced mostly nonseptate or two-celled conidia. Conidia produced by the con 4 and $\operatorname{con} 7$ mutants also are morphologically different from those of the coml mutants (Odenbach et al. 2007; Shi and Leung 1995). Therefore, it is unlikely for these genes to be functionally related to COM1.

In Saccharomyces cerevisiae, the nonhinstone proteins Nhp6A and Nhp6B were reported to facilitate global transcriptional regulation and to play an important role in the growth and morphology (Costigan et al. 1994). Null mutants of MNH6 in $M$. oryzae, the homolog of $N H P 6 A / B$, exhibited pleiotropic effects on fungal morphogenesis, including reduction in hyphal growth, conidiation, appressorial penetration, and infectious growth in host cells (Lu et al. 2007). Phenotypically, the $\Delta$ coml mutant is similar to the $\Delta m n h 6$ mutant. However, the $\Delta m n h 6$ mutant, unlike the $\Delta$ coml mutant, had a reduced growth rate. The eGFP-Mnh6 fusion proteins localized to the nucleus (Liu et al. 2007). It is possible that MNH6 and COMI are functionally related in regulating conidium development and other processes. However, the relationship between these two genes remains to be characterized.

Another interesting observation is that conidia of the $\Delta \mathrm{coml}$ mutant are reduced in their sedimentation rate (Fig. 6). Although changes in conidium morphology may be directly responsible for the reduction in the sedimentation rate, it remains possible that the density of cytoplasm is reduced in the $\Delta$ coml mutant. In nature, conidia of $M$. oryzae are dispersed by rain splashes or wind. Whether changes in the sedimentation rate would affect the dispersal and attachment of conidia and, ultimately, their virulence under field conditions remains to be tested.

Recently, we have isolated an $F$. graminearum mutant deleted in the COM1 ortholog. Preliminary studies have indicated that the $\Delta$ fgcom 1 mutant also is defective in conidium mor-

Table 3. Polymerase chain reaction primers used in this study

\begin{tabular}{ll}
\hline Primer & \multicolumn{1}{c}{ Sequence $\left(\mathbf{5}^{\prime} \mathbf{-} \mathbf{3}^{\prime}\right)$} \\
\hline P1 & CAGAGTGGCAGAAGCTGTTG \\
P2 & TGTAGTATGCGTCAGCCTTG \\
P3 & GAAGACGAGGTCGAGGGTGG \\
P4 & GACAGACGTCGCGGTGAGTT \\
P5 & TCTGGACCGATGGCTGTGTAG \\
P6 & CACCACTGCGCGTCGTACTC \\
P7 & ATTACTCGAGATCCGTAGATCCCTG \\
P8 & ATATGTCGACCTTGATGGGCAACTC \\
P9 & ATTACTGCAGATCCGTAGATCCCTG \\
P10 & ATATGGATCCTCTCCTAGTGGGACTG \\
Lf & GAACCCGGGACTACATGAAAACCAAC \\
Lr & CACGAAGCTTCAAAGAGGCGTACTC \\
Rf & TTACTCGAGACATCTTGATGGGCAAC \\
Rr & ATAGGGCCCGGTACTGGGTACAAAG \\
dw2f & AATCGATATGTCGTTAGCCAGG \\
dw2r & TAAGCTTGGAAGCATTGGTTG \\
dw21f & ATATACTAGTGCAGTCGAAAAGAAAC \\
dw21r & TAATACTAGTGTTTGTGAGCTCGAC \\
dw22f & ATATACTAGTGAGGAGGAGGACAGC \\
dw22r & TAATACTAGTCTTTGCTGTGATCTG \\
dw23f & ATATACTAGTGTCAAGGAGCCAGTG \\
dw23r & TAATACTAGTTGTGCCCGAGCTGGA \\
\hline
\end{tabular}

phology and plant infection (unpublished data). It is likely that COM1 and its orthologs are a part of a well-conserved regulatory network involved in conidium development and invasive growth in $M$. oryzae and other fungal pathogens. Because conidia play a critical role in pathogen dispersal and initiation of plant infection, it will be important to identify genes that are functionally related to or regulated by COM1 in M. oryzae and other fungi.

\section{MATERIALS AND METHODS}

\section{Strains and growth conditions.}

The $M$. oryzae field isolate $\mathrm{P} 131$ and various transformants generated in this study were cultured at $25^{\circ} \mathrm{C}$ on OTA plates as described by Peng and Shishiyama (1988). Mycelia collected from 2-day-old cultures in complete medium $(0.6 \%$ yeast extract, $0.3 \%$ acid casein hydrolysate, $0.3 \%$ enzymic casein hydrolysate, and $1 \%$ sucrose) shaken at $150 \mathrm{rpm}$ were used for the isolation of fungal DNA and protoplasts. Protoplasts were isolated and transformed with the PEG method as described by Park and associates (2006). Media were supplemented with hygromycin B at $250 \mu \mathrm{g} / \mathrm{ml}$ (Roche, Branchburg, NJ, U.S.A.) or neomycin at $400 \mu \mathrm{g} / \mathrm{ml}$ (Ameresco, Solon, OH, U.S.A.) in order to select hygromycin-resistant or neomycin-resistant transformants, respectively. Conidia were harvested from 10-dayold OTA cultures to assay conidiation and measure conidium morphology.

\section{Molecular manipulations with DNA and RNA.}

Genomic DNAs were extracted from vegetative hyphae with the cetyltrimethylammonium bromide protocol (Xu and Hamer 1996). RNA was extracted using the Trizol kit (SBS Company, Beijing). Standard molecular biology procedures were followed for plasmid isolation, DNA and RNA gel blot analyses, and enzymatic manipulation with DNA (Sambrook and Russell 2001). Probes were labeled with the Random Primer Labeling Kit (Takara, Dalian, China). Plasmid constructs were sequenced with an ABI 3730 sequencer (SunBio Company, Beijing). BLAST programs were used to search for homologous DNA and protein sequences. Protein sequence comparison and alignment analyses were performed with the ClustalW, ClustalX, and PhyloDraw programs.

\section{Appressorium formation, penetration, and cytorrhysis assays.}

Conidia harvested from OTA plates were resuspended in sterile distilled water to a concentration of $5 \times 10^{5}$ conidia $/ \mathrm{ml}$. Conidial suspension droplets ( 3 to $5 \mu$ l containing approximately 2,000 conidia) were placed on onion (Allium cepa) epidermal cells and incubated in a moist chamber at $25^{\circ} \mathrm{C}$. Conidium germination, appressorium formation, and infectious hyphal growth were examined at 2, 24 and $36 \mathrm{~h}$ postinoculation, respectively, with a Nikon Ni90 microscope (Nikon, Japan) as described by Xue and associates (2002). Mature appressoria formed on plastic cover surfaces for $24 \mathrm{~h}$ were used for cytorrhysis assays, as described by Howard and associates (1991).

\section{Infection assays.}

M. grisea conidia were collected from 7-day-old OTA plates and resuspended to $2 \times 10^{4}$ conidia/ml in a $0.025 \%$ Tween-20 solution. Four-week-old seedlings of rice (Oryzae sativa) cv. Lijiangxintuanheigu and 8-day-old seedlings of barley (Hordeum vulgare) cv. E9 were used for spray infection assays (Peng and Shishiyama 1988; Park et al. 2004). Plant incubation was performed as described previously (Peng and Shishiyama 1988; Xu et al. 1997). Lesion formation was examined 7 days 
postinoculation. The mean number of lesions formed on $5-\mathrm{cm}$ leaf tips was determined as described previously (Peng and Shishiyama 1988).

\section{Identification and characterization of the $\Delta$ com 1 disruption mutants.}

For generating REMI transformants, the plasmid pUCATPH (Lu et al. 1994) was linearized by HindIII, KpnI, SmaI, or XhoI and transformed into P131 protoplasts. The copy numbers of transforming vector integrations into the genome were analyzed by genomic Southern blotting. In a screen for mutants defective in conidium morphology, five transformants (K2877, S1005, H3035, H3587, and X54) were found to produce similar elongated, slender conidia. The mutant H3035 was generated by transformation with HindIII-digested pUCATPH, which lacked an EcoRV site. Genomic DNA of H3035 was digested with $E c o \mathrm{RV}$, circularized by self-ligation, and transformed into Escherichia coli JM109. A plasmid pHEv8 containing a 2.2-kb fragment of the P131 genomic DNA was isolated and sequenced. The same approach was used to obtain the flanking sequences of the transforming vector from the mutants $\mathrm{H} 3587$ (EcoRV digestion) and X54 (BamHI digestion).

\section{Generation of the $C O M 1$ gene replacement construct and mutant.}

For generating the COM1 gene replacement vector pCOM12.4 , the $1.0-\mathrm{kb}$ upstream and $1.0-\mathrm{kb}$ downstream sequences of the COM1 gene were amplified with primer pairs $\mathrm{Lf} / \mathrm{Lr}$ and $\mathrm{Rf} / \mathrm{Rr}$, respectively. The resulting PCR products were cloned into the SmaI-HindIII and XhoI-ApaI sites of pKNH, respectively. The vector $\mathrm{pKNH}$ was generated by subcloning a $2.1-\mathrm{kb}$ SalI fragment with the bacterial HPT gene from the plasmid pUCATPH into pKN (Lu et al. 1994; Khang et al. 2005) to provide the neomycin resistance (NPTII) gene. After linearization with NotI, pCOM1-2.4 was transformed into P131. Hygromycin-resistant transformants were isolated and assayed for resistance to neomycin and screened by PCR. The primer pairs P3/P4 and P5/P6 (Fig. 3A; Table 3) were used to amplify $1.9-$ and $1.6-\mathrm{kb}$ fragments, respectively, that are diagnostic for homologous recombination events at the upstream and downstream flanking sequences. Primer pair P1/P2 (Table 3) amplified a $1.0-\mathrm{kb}$ fragment in the wild-type but not in the $\Delta$ com 1 mutant. Putative $\Delta$ com 1 mutants were mapped in more detail by Southern blot analyses.

\section{Complementation of the $\Delta \operatorname{com} 1$ mutant with the wild-type $C O M 1$ allele.}

For complementation assays, the COM1 gene, along with $1.0-\mathrm{kb}$ upstream and $0.5-\mathrm{kb}$ downstream sequences, was amplified with primers $\mathrm{P} 9$ and $\mathrm{P} 10$ and cloned between the Pst I and BamHI sites in $\mathrm{pKN}$ to generate $\mathrm{pKNS} 2$. After Not I digestion, pKNS2 was transformed into the coml mutants. The resulting neomycin-resistant transformants were confirmed by Southern analysis and analyzed for conidium morphology, appressorium formation, and infectious hyphal growth on onion epidermal cells and virulence on rice seedlings.

\section{Microscopic examination of conidium morphology.}

Freshly harvested conidia were examined under a Nikon Ni90 microscope and measured for their length and width. For TEM examination, conidia were washed with $3 \%$ (vol/vol) glutaraldehyde and embedded in $2 \%$ agar. After being fixed with $3 \%$ (vol/vol) glutaraldehyde in $50 \mathrm{mM}$ phosphate buffer (pH 6.8) for 3 to $6 \mathrm{~h}$ at $4^{\circ} \mathrm{C}$, conidium blocks were sliced into small pieces, rinsed with $50 \mathrm{mM}$ phosphate buffer, and postfixed with $1 \%$ (wt/vol) osmium tetroxide in the same buffer for $2 \mathrm{~h}$ at $4^{\circ} \mathrm{C}$. The samples were then dehydrated in a graded ethanol series, embedded in LR White (TAAB Laboratories, Munich) and polymerized at $50^{\circ} \mathrm{C}$ for 1 day. Ultrathin sections were prepared with a diamond knife and collected on 200mesh copper grids. After contrasting with $1 \%$ (wt/vol) uranyl acetate and lead citrate, the grids were examined with a ZeissEM10 electron microscope at $80 \mathrm{kV}$ (Kang et al. 2005).

\section{Generation of eGFP fusion constructs.}

The COM1-eGFP fusion vector pKG31 was generated by cloning the COM1 cDNA into pKNTG, which was constructed by cloning the $e G F P-\operatorname{Tr} p \mathrm{C}$ terminator sequence into $\mathrm{pKN}$ (Khang et al. 2005). The eGFP-COM1 fusion construct, pKG32, was generated by cloning the COM1 ORF between $e G F P$ and the $\operatorname{Trp} C$ terminator. Both vectors contained the eGFP fusion under the control of the COMI promoter and were transformed into the $\Delta \mathrm{coml}$ mutant kc48.

The $C O M 1^{335-613}$-eGFP fusion was constructed by cloning a fragment amplified with the primer pair dw2f/dw2r from pKG31 into pKNTGRP27 as pDWCOM1-2. The plasmid pKNTGRP27 was generated by cloning the strong, constitutive RP27 promoter into pKNTG (Bourett et al. 2002). Plasmids pDWCOM1-21, pDWCOM1-22, and pDWCOM1-23 deleted of different NLS sequences were generated by self-ligation of PCR products amplified from pDWCOM1-2 with primer pairs dw21f/dw21r, dw22f/dw22r, and dw23f/dw23r, respectively. After NotI digestion, all constructs were transformed into the wild-type P131. Plasmid pKG31-NLS2 carrying the $C O M 1^{\triangle P P V K R P R E}$-eGFP construct was generated by self-ligation of PCR products amplified from pKG31 with primer pair dw22f/dw22r. After NotI digestion, pKG31-NLS2 was transformed into the $\Delta$ com 1 mutant $\mathrm{kc} 48$. To generate the $\mathrm{pECOM} 1$ construct, the 1.1-kb promoter region of COM1 was amplified with the primer pair P7/P8 (Table 3) and cloned between the XhoI-SalI sites of pKNTG. The resulting construct, pECOM1, was transformed into P131.

\section{Sedimentation velocity tests.}

Conidia harvested from 10-day-old cultures of the wild-type and $\Delta$ com 1 mutant strains were resuspended to $2 \times 10^{6}$ co$\mathrm{nidia} / \mathrm{ml}$ in distilled water. An aliquot of $0.5 \mathrm{ml}$ of conidium suspension was gently loaded on the top of an 80-cm-long, 1.6-cm-diameter column filled with sterile distilled water. The column was then capped and connected to an HL-2 constant flow pump (Huxi Instrument Company, Shanghai, China). After $10 \mathrm{~min}$ of sedimentation, conidial samples were collected from the column at a constant speed of $0.67 \mathrm{ml} / \mathrm{min}$. Each 2-ml sample was collected separately and the total number of conidia in 3- $\mu$ l samples of each fraction was determined.

\section{ACKNOWLEDGMENTS}

This manuscript was supported by the 973 program (grant number 2006CB101901) and the 863 program (grant number 2006AA10A201) to Y.-L. Peng from the Ministry of Sciences and Technology, China, and was selected as a feature article through the APS/CSPP Cooperative Agreement. We thank A. Jackson at the University of California-Berkeley for critical reading of this article, M. Xue and F. He at China Agricultural University for assistance with bioinformatic analysis, and $\mathrm{S}$. Wei for constructing the REMI mutant library and providing the mutant X54.

\section{LITERATURE CITED}

Bourett, T. M., Sweigard, J. A., Czymmek, K. J., Carroll, A., and Howard, R. J. 2002. Reef coral fluorescent proteins for visualizing fungal pathogens. Fungal Genet. Biol. 37: 211-220.

Busby, T. M., Miller, K. Y., and Miller, B. L. 1996. Suppression and enhancement of the Aspergillus nidulans medusa mutation by altered dosage of the bristle and stunted genes. Genetics 143:155-163.

Costigan, C., Kolodrubetz, D., and Snyder, M. 1994. NHP6A and NHP6B, 
which encode HMG1-like proteins, are candidates for downstream components of the yeast SLT2 mitogen-activated protein kinase pathway. Mol. Cell Biol. 14:2391-2403.

Dean, R. A., Talbot, N. J., Ebbole, D. J., Farman, M. L., Mitchell, T. K., Orbach, M. J., Thon, M., Kulkarni, R., Xu, J. R., Pan, H. Q., Read, N. D., Lee, Y. H., Carbone, I., Brown, D., Oh, Y. Y., Donofrio, N., Jeong, J. S., Soanes, D. M., Djonovic, S., Kolomiets, E., Rehmeyer, C., Li, W. X., Hardling, M., Kim, S., Lebrun, M. H., Bohnert, H., Coughlan, S., Butler, J., Calvo, S., Ma, L. J., Nicol, R., Purcell, S., Nusbaum, C., Galagan, J. E., and Birren, B. W. 2005. The genome sequence of the rice blast fungus Magnaporthe oryzae. Nature 434:980-986.

de Jong, J. C., McCormack, B. J., Smirnoff, N., and Talbot, N. J. 1997.Glycerol generates turgor in rice blast. Nature 389:244-245.

Ford, T. L., Cooley, J. T., and Christou, P. 1994. Current status for gene transfer into rice utilizing variety-independent delivery systems. Pages 195-208 in: Rice Blast Disease. CAB International, Wallingford, U.K.

Guptaa, A., and Chattoo, B. B. 2007. A novel gene MGA1 is required for appressorium formation in Magnaporthe oryzae. Fungal Genet. Biol. 44:1157-1169.

Guptaa, A., and Chattoo, B. B. 2008. Functional analysis of a novel ABC transporter ABC4 from Magnaporthe oryzae. FEMS (Fed. Eur. Microbiol. Soc.) Microbiol. Lett. 278:22-28.

Hamer, J. E., Howard, R. J., Chumley, F. G., and Valent, B. 1988. A mechanism for surface attachment of spores of a plant pathogenic fungus. Science 239:288-290.

Hamer, J. E., Valent, B., and Chumley, F. G. 1989. Mutations at the Smo genetic locus affect the shape of diverse cell types in the rice blast fungus. Genetics 122:351-361.

Howard, R. J., Ferrari, M. A., Roach, D. H., and Money, N. P. 1991. Penetration of hard substrates by a fungus employing enormous turgor pressures. Proc. Natl. Acad. Sci. U.S.A. 88:11281-11284.

Jeon, J., Goh, J., Yoo, S., Chi, M. H., Choi, J., Rho ,H. S., Park, J., Han, S. S., Kim, B. R., Park, S. Y., Kim, S., Lee, Y. H. 2008. A putative MAP kinase kinase kinase, MCK1, is required for cell wall integrity and pathogenicity of the rice blast fungus, Magnaporthe oryzae. Mol. PlantMicrobe Interact. 21:525-534.

Kankanala, P., Czymmek, K., and Valent, B. 2007. Roles for rice membrane dynamics and plasmodesmata during biotrophic invasion by the blast fungus. Plant Cell 19:706-724.

Khang, C. H., Park, S. Y., Lee, Y. H., and Kang, S. C. 2005. A dual selection based, targeted gene replacement tool for Magnaporthe oryzae and Fusarium oxysporum. Fungal Genet. Biol. 42:483-492

Lau, G. W., and Hamer, J. E. 1998. Acropetal: a genetic locus required for conidiophore architecture and pathogenicity in the rice blast fungus. Fungal Genet. Biol. 24:228-239.

Li, L., Xue, C. Y., Bruno, K., Nishimura, M., and Xu, J. R. 2004. Two PKA kinase genes, CHM1 and MST20, have distinct functions in Magnaporthe oryzae. Mol. Plant-Microbe Interact. 17:547-556.

Liu, X. H., Lu, J. P., Zhang, L., Dong, B., Min, H., and Lin, F. C. 2007. Involvement of a Magnaporthe grisea serine/threonine kinase gene, $M g A T G 1$, in appressorium turgor and pathogenesis. Eukaryotic Cell 6:997-1005

Lu, J. P., Feng, X. X., Liu, X. H., Lu, Q., Wang, H. K., and Lin, F. C. 2007. Mnh6, a histone protein, is required for fungal development and pathogenicity of Magnaporthe grisea. Fungal Genet. Biol. 44:819-829.

Lu, S., Lyngholm, L., Yang, G., Bronson, C., Yoder, O. C., and Turgeon, B. G. 1994. Tagged mutations at the Tox1 locus of Cochliobolus heterostrophus by restriction enzyme-mediated integration. Proc. Natl. Acad. Sci. U.S.A. 91:12649-12653.
Massari, M. E., and Murre, C. 2000. Helix-loop-helix proteins: regulators of transcription in eukaryotic organisms. Mol. Cell Biol. 20:429-440.

Nishimura, M., Hayashi, N., Jwa, N. S., Lau, G. W., Hamer, J. E., and Hasebe, A. 2000. Insertion of the LINE retrotransposon MGL causes a conidiophore pattern mutation in Magnaporthe grisea. Mol. PlantMicrobe Interact. 13:892-894.

Odenbach, D., Breth, B., Thines, E., Weber, R. W., Anke, H., and Foster, A. J. 2007. The transcription factor Con $7 p$ is a central regulator of infection-related morphogenesis in the rice blast fungus Magnaporthe oryzae. Mol. Microbiol. 64:293-307.

Park, G., Bruno, K. S., Staiger, C. J., Talbot, N. J., and Xu, J. R. 2004. Independent genetic mechanisms mediate turgor generation and penetration peg formation during plant infection in the rice blast fungus. Mol. Microbiol. 53:1695-1707.

Park, G., Xue, C. Y., Zhao, X. H., Kim, Y., Orbach, M., and Xu, J. R. 2006. Multiple upstream signals converge on an adaptor protein Mst50 to activate the PMK1 pathway in Magnaporthe grisea. Plant Cell 18:28222835.

Peng, Y., and Shishiyama, J. 1988. Temporal sequence of cytological events in rice leaves infected with Pyricularia oryzae. Can. J. Bot. 66:730-735.

Pinnschmidt, H. O., Bonman, J. M., and Kranz, J. 1995. Lesion development and sporulation of rice blast. J. Plant Dis. Prot. 102:299-306.

Sambrook, J., and Russell, D. W. 2001. Molecular Cloning: A Laboratory Manual. Cold Spring Harbor Laboratory Press, Cold Spring Harbor, NY, U.S.A.

Shi, Z., and Leung, H. 1995. Genetic analysis of sporulation in Magnaporthe oryzae by chemical and insertional mutagenesis. Mol. PlantMicrobe Interact. 8:949-959.

Soanes, D. M., and Talbot, N. J. 2005. A bioinformatic tool for analysis of EST transcript abundance during infection-related development by Magnaporthe oryzae. Mol. Plant Pathol. 6:503-512.

Sweigard, J. A., Carroll, A. M., Farrall, L., Chumley, F. G., and Valent, B. 1998. Magnaporthe oryzae pathogenicity genes obtained through insertional mutagenesis. Mol. Plant-Microbe Interact. 11:404-412.

Talbot, N. J. 2003. On the trail of a cereal killer: exploring the biology of Magnaporthe oryzae. Annu. Rev. Microbiol. 57:177-202.

Valent, B. 1990. Rice blast as a model system for plant pathology. Phytopathology 80:33-36.

Valent, B., Farrall, L., and Chumley, F.G. 1991. Magnaporthe oryzae genes for pathogenicity and virulence identified through a series of backcrosses. Genetics 127:87-101.

$\mathrm{Xu}$, J. R., and Hamer, J. E. 1996. MAP kinase and cAMP signaling regulate infection structure formation and pathogenic growth in the rice blast fungus Magnaporthe oryzae. Genes Dev. 10:2696-2706.

Xu, J. R., Urban, M., Sweigard, J. A., and Hamer, J. E. 1997. The CPKA gene of Magnaporthe oryzae is essential for appressorial penetration. Mol. Plant-Microbe Interact. 10:187-194.

Xu, J. R., Zhao, X. H., Dean, R.A. 2007. From genes to genomics: a new paradigm for studying fungal pathogenesis in Magnaporthe oryzae. Adv. Genet. 57:175-218.

Yu, J. H., Mah, J. H., and Seo, J. A. 2006. Growth and developmental control in the model and pathogenic aspergilli. Eukaryot. Cell 5:1577-1584.

\section{AUTHOR-RECOMMENDED INTERNET RESOURCES}

Scratch Protein Predictor (SSpro): scratch.proteomics.ics.uci.edu/ PSORTII server: psort.ims.u-tokyo.ac.jp

PredictNLS server: cubic.bioc.columbia.edu/predictNLS

A change was made in the Acknowledgments section on January 7, 2010. 